\title{
High frequency response of LWIR HgCdTe photodiodes operated under zero-bias mode
}

\author{
M. Kopytko' ${ }^{1}$ P. Martyniuk ${ }^{1} \cdot$ P. Madejczyk ${ }^{1}$ - K. Jóźwikowski ${ }^{1} \cdot$ \\ J. Rutkowski ${ }^{1}$
}

Received: 4 August 2017/Accepted: 15 January 2018/Published online: 18 January 2018

(C) The Author(s) 2018. This article is an open access publication

\begin{abstract}
High frequency response is an important capability of infrared detectors in many applications. High-temperature long wavelength infrared $\mathrm{HgCdTe}$ heterostructure photodiodes exhibit sub-nanosecond time constants while operating under reverse bias. However, the noise, as well as the high current requirements are severe obstacles to their widespread applications. Thus, the present efforts are focused on a zero-bias operation of infrared detectors. A numerical modelling was used for investigation of the device design on the response time and current responsivity of $\mathrm{HgCdTe}$ photodiodes operating at $200 \mathrm{~K}$ and zero-bias mode. A formulation of the equations for carrier transport in semiconductors is presented in the Fourier space method in order to analyze spectrum characteristic of currents generated by harmonic optical signals. The method is valid in describing the high frequency response of IR devices based on $\mathrm{HgCdTe}$ multilayer heterostructures.
\end{abstract}

Keywords HgCdTe photodiodes · Photodiode response - Time constant

\section{Introduction}

High-operating temperature (HOT) photodetectors is one of the hot topics in infrared (IR) technology for the middle (MWIR-3-5 $\mu \mathrm{m}$ ), as well as the long wavelength (LWIR8-12 $\mu \mathrm{m}$ ) IR range (Piotrowski and Rogalski 2007; Kinch 2015). During the last three

Topical Collection on Numerical Simulation of Optoelectronic Devices, NUSOD’ 17

Guest Edited by Matthias Auf der Maur, Weida Hu, Slawomir Sujecki, Yuh-Renn Wu, Niels Gregersen, Paolo Bardella

M. Kopytko

malgorzata.kopytko@wat.edu.pl

1 Institute of Applied Physics, Military University of Technology, 2 Urbanowicza St, 00-908 Warsaw, Poland 
decades, HOT detectors based on $\mathrm{HgCdTe}$ heterostructures have been developed in Poland with a great emphasis on its frequency response (Piotrowski et al. 2010; Martyniuk et al. 2016; Madejczyk et al. 2017; Grodecki et al. 2017). Fast response of IR detectors is an important property which determines numerous practical applications, such as long-range free space communications, ultrafast spectroscopy, gas leak detection in civilian applications or weapon control and missile guidance systems in military applications.

In semiconductor devices, high frequency response can be achieved by a fast decay of photogenerated carriers due to rapid recombination in the absorber region, or by a fast transport of photogenerated carriers to contacts. First method is used in many fast response devices including photoconductors. However, this choice is not optimal for HOT detectors. Recombination mechanisms as a statistical processes lead to poor signal-to-noise performance and only the second solution finds practical application for heterostructure photodiodes.

In this case, a special $\mathrm{N}^{+}-\mathrm{p}-\mathrm{P}^{+}$heterostructure design has been developed, where $p$-type absorber is an advantageous material for the fast response of $\mathrm{HgCdTe}$ detectors. The $\mathrm{N}^{+}$-p$\mathrm{P}^{+}$devices with combination of exclusion and extraction junctions have demonstrated suppression of Auger generation-recombination (GR) mechanisms by reducing the absorber carrier density below thermal equilibrium in reverse bias condition (Elliott et al. 1996; Ashley and Elliott 1985; Ashley et al. 1985; Piotrowski 1995; Jakšić and Djurić 2004; Emelie et al. 2007, 2008; Velicu et al. 2010; Kopytko et al. 2016). The exclusion effect is limited by the level of acceptor concentration (electrical carrier neutrality). By applying a low doping semiconductor, so that doping concentration should be considerable lower than intrinsic concentration in a device operating temperature, it is possible to achieve a background limited performance (BLIP condition) with thermoelectric cooling and even at room temperature. $\mathrm{HgCdTe}$ heterostructure photodiodes also exhibit subnanosecond time constants while operate under reverse bias (Madejczyk et al. 2017; Grodecki et al. 2017). However, non-equilibrium conditions lead to an excessive low frequency $1 / f$ noise that extends up to $\mathrm{MHz}$ range (Ciura et al. 2016).

Thus, present efforts are focused on a zero-bias operation. Unfortunately, the time constants of unbiased detectors are still at the level of several ns. In this case, the response time is mostly determined by ambipoloar diffusion of photogenerated charge carriers to contacts, as well as $R C$ constant mainly limited by series resistance. To reduce the equivalent $R C$ time constant of photodiode, transimpedance preamplifiers with a low input resistance should be used. However, device architecture still require optimization to improve the diffusion transit time.

In this paper, we discuss a possible configurations of LWIR $\mathrm{N}^{+}-\mathrm{p}-\mathrm{P}^{+} \mathrm{Hg}_{1-x} \mathrm{Cd}_{x} \mathrm{Te}$ photodiodes and report on a dependence of the response properties on the thickness, and doping of the absorbing layer. Moreover, the influence of graded Cd alloy composition in the absorber layer on device properties is investigated.

\section{Method of analysis}

Our computer software based on a solution of the set of transport equations consisting of the continuity equations for electrons, holes, the Poisson's equation, and the thermal conductivity equation given by Van Roosbroeck (1950) and Kurata (1982): 


$$
\begin{gathered}
\frac{\partial n}{\partial t}=\frac{1}{q} \nabla \vec{j}_{n}+(G-R), \\
\frac{\partial p}{\partial t}=-\frac{1}{q} \nabla \vec{j}_{p}+(G-R), \\
\nabla^{2} \Psi=-\frac{q}{\varepsilon \varepsilon_{0}}\left[N_{D}^{+}-N_{A}^{-}+p-n\right]-\frac{1}{\varepsilon} \nabla \Psi \nabla \varepsilon, \\
C_{V} \frac{\partial T}{\partial t}-H=\nabla(\chi \cdot \nabla T),
\end{gathered}
$$

where $\Psi$ is the electrostatic potential, $j$ is current density, $q$ is elementary charge, $\varepsilon_{0}$ is the vacuum permittivity, $\varepsilon$ is relative permittivity, $C_{v}$ is specific heat, $\chi$ thermal conductivity coefficient, $H$ is the rate of heat generation per volume unit, $T$ temperature, $t$ is the time and $\nabla$ is the gradient. $n$ and $p$ denote electron and hole, respectively. $N_{A}^{+}$and $N_{D}^{-}$are concentrations of ionized acceptors and donors, respectively. The difference $(G-R)$ is the net generation of electron-hole pairs, and depends on all generation-recombination (GR) mechanisms including influence of thermal mechanisms (Shockley-Read-Hall (SRH), Auger, Radiative) as well as tunnelling mechanisms due to band-to-band tunnelling (BTB) and trap-assisted tunnelling (TAT). In some cases, the radiative GR process can be ignored because photon recycling restricts the influence of that process on the performance of HgCdTe photodiodes (Humpreys 1986; Jóźwikowski et al. 2011; Jóźwikowski et al. 2012).

Electron and hole current densities are usually expressed as

$$
\begin{aligned}
& \vec{j}_{n}=q \mu_{n} n E+q D_{e} \nabla n, \\
& \vec{j}_{p}=q \mu_{p} p E-q D_{h} \nabla p,
\end{aligned}
$$

where $E$ is the electric field, $\mu_{e}$ and $\mu_{h}$ are the electron and hole mobility, $D_{e}$ and $D_{h}$ are the diffusion coefficients for electrons and holes.

The numerical method applied in this study employs Newton's algorithm to obtain a steady-state solution to the set of transport Eqs. (1)-(4), using an initial distribution of the electrostatic potential in thermodynamic equilibrium which is obtained from conditions of electrical neutrality. Poisson's equation under equilibrium conditions gives the first trial function in the iterative procedure that leads to the solution of the set of transport equations under nonequilibrium conditions. Stabilized and fully controllable numerical algorithms to solve transport equations in complex heterostructure have been elaborated in Ref. (Jóźwikowska 2008).

Next, to obtain a time variation of photocurrent after switching the optical signal, we have adopted the Fourier expansion method (Kopytko et al. 2010, 2013). Having the stationary solution of Eqs. (1)-(4) in a steady state condition, a photodiode is illuminated by small optical signal. The time variable flux is defined as a harmonic function expressed as

$$
\Phi(t)=\Phi_{0} \exp (i \omega t)
$$

where $\Phi_{0}$ is the amplitude and indicates the imaginary part, $\omega$ is the frequency, and $t$ is the time. Under illumination, all photoelectric parameters of the heterostructure have changed. Example, the change of electrons concentration can be expressed as 


$$
\Delta n(\vec{r}, t)=n(\vec{r}, t)-n_{0}(\vec{r})=n_{\omega}(\vec{r}) e^{i \omega t},
$$

where $n_{0}$ is the concentration in steady state condition and $n_{\omega}(\vec{r})$ is the complex amplitude of the concentration change. On this basis we can calculate the complex amplitudes of other parameters (holes concentration, the electrostatic potential, quasi Fermi levels for electrons and holes, and temperature) for the definite frequency $\omega$ to obtain the complex amplitude of photocurrent. The real part of the obtained photocurrent can be approximated by equation

$$
J_{\Phi}=\frac{J_{0}}{\left(1+\omega^{2} t^{2}\right)} .
$$

$J_{0}$ is the amplitude of ac current. In the simplest detector's case, the time constant $\tau$ defined by the cut-off frequency $f_{T}$ at $-3 \mathrm{~dB}$ line can be calculated as:

$$
\tau=\frac{1}{2 \pi f_{T(-3 d B)}} .
$$

There are more complex cases if the detector time constant is limited by different mechanisms such as different carrier recombination mechanisms, space charge and diffusion capacitance (Phillips and Gordon 1996).

In calculations, the wavelength of the incident light was assumed as $\lambda_{\text {inc }}=9 \mu \mathrm{m}$ and the power density as $P_{\text {inc }}=0.1 \mathrm{~W} / \mathrm{cm}^{2}$. Numerical analysis of the frequency response of

\begin{tabular}{|c|c|c|c|c|}
\hline & & $t[\mu \mathrm{m}]$ & $x$ & $N_{A}, N_{D}\left[\mathrm{~cm}^{-3}\right]$ \\
\hline Cap contact layer & $\mathrm{P}^{+}$ & 2.5 & 0.34 & $5 \times 10^{17}$ \\
\hline \multirow{3}{*}{ Absorber } & \multirow{3}{*}{$p$} & \multicolumn{3}{|c|}{ Graded interface } \\
\hline & & Variable & $\begin{array}{c}0.19 \\
0.18 \rightarrow 0.2\end{array}$ & Variable \\
\hline & & \multicolumn{3}{|c|}{ Graded interface } \\
\hline Bottom contact layer & $\mathrm{N}^{+}$ & 6.5 & 0.34 & $5 \times 10^{17}$ \\
\hline Substrate & & & & \\
\hline & & & & \\
\hline
\end{tabular}
the photodiode shows a slightly different approach than the one performed in the time domain (Pawluczyk et al. 2015).

Fig. $1 \mathrm{P}^{+}-\mathrm{p}-\mathrm{N}^{+}$diode architecture and structural parameters taken in modeling 


\section{Devices design}

The investigated device is a photodiode based on $\mathrm{Hg}_{1-x} \mathrm{Cd}_{x} \mathrm{Te}$ multilayer heterostructures. Figure 1 shows schematic cross-sections of $\mathrm{N}^{+}-\mathrm{p}-\mathrm{P}^{+}$mesa-structure photodiode. Composition, doping level and thickness of the absorber region is optimized for the best compromise between requirements of efficient use of IR radiation and low thermal generation. The absorber layer is surrounded by a wider bandgap, highly doped contact layers to minimize thermal generation and parasitic impedance. Graded band-gap interfaces represent the $x$-graded regions created by interdiffusion processes during the metal organic chemical vapour deposition (MOCVD) growth at $350{ }^{\circ} \mathrm{C}$. What more, gradients can be specially introduced to suppress tunnelling currents under a reverse bias. The device is backside illuminated through the $\mathrm{N}^{+}$layer that plays the role of an infrared transmitting window for photons with energies below the band gap. Since a diffusion length in the $\mathrm{N}^{+}$ layer is short compared to its thickness, the charge carriers generated in the layer do not produce photocurrent. The band gap of the layer determines the cut-on wavelength of the device.

In the paper we have analysed two possible configurations of $\mathrm{N}^{+}$-p- $\mathrm{P}^{+} \mathrm{Hg}_{1-x} \mathrm{Cd}_{x} \mathrm{Te}$ photodiodes:

- with a constant $\mathrm{Cd}$ alloy composition in the absorber layer, $x_{A b s}=0.19$ was chosen for $11.6 \mu \mathrm{m}$ cut-off wavelength at $200 \mathrm{~K}$, and

- with a graded composition in the absorber layer, ranges from 0.18 to 0.20 , and adjusted to maintain a constant peak wavelength (about $9 \mu \mathrm{m}$ ) at a temperature of operation in comparison to the device with constant composition.

Structural parameters such as absorber doping and thickness were changed in calculations to obtain a trade-off between contradictory requirements of reaching high current responsivity and fast response time under zero-bias conditions. In order to show the influence of the doping concentration of the absorber on the detectors performances, calculations have been done for and a low $\left(N_{A}=0.1 n_{i}\right)$ and a high $\left(N_{A}=10 n_{i}\right)$ doping level of the absorbing layer. $n_{i}$ is the intrinsic carrier concentration in semiconductor at a given temperature.

The assumed composition and doping concentration profiles of $\mathrm{N}^{+}-\mathrm{p}-\mathrm{P}^{+} \mathrm{HgCdTe}$ photodiodes permit us to calculate the energy band diagrams. Figure 2 shows the simulated energy band diagrams for unbiased photodiodes with a constant and graded $\mathrm{Cd}$ alloy composition in the absorber layer, respectively. In our calculations, the operation temperature was chosen at $200 \mathrm{~K}$. In this case, the intrinsic carrier concentration is of about $10^{16} \mathrm{~cm} \mathrm{~cm}^{-3} .5-\mu \mathrm{m}$ thick absorber was chosen as a typical thickness that corresponds to the ambipolar diffusion length. As we can see, doping level has the important effect of shifting the energy bands relative to the Fermi level $\left(E_{F}\right)$. For low levels of doping, the Fermi level lies close to the edge of the conduction band $\left(E_{C}\right)$. For higher doping, the Fermi level moves toward the middle of the bandgap.

\section{Results and discussion}

The transport of photogenerated carriers to contacts in complex heterostructure devices is determined by two factors:

- the drift transit time, $\tau_{d r}$, across the space charge regions; 

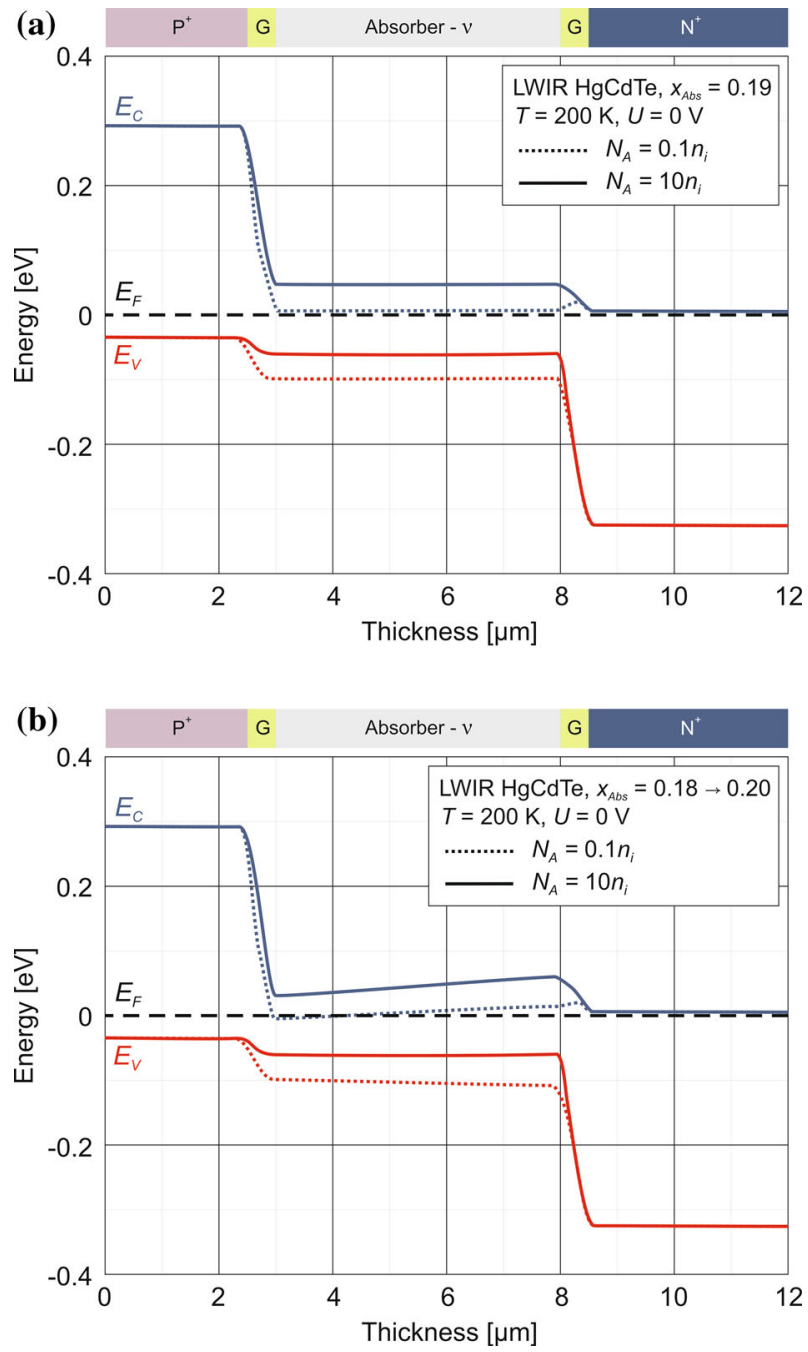

Fig. 2 Calculated band diagram for LWIR $\mathrm{N}^{+}-\mathrm{p}-\mathrm{P}^{+} \mathrm{HgCdTe}$ photodiode with a constant (a) and graded (b) $\mathrm{Cd}$ alloy composition in the absorber layer

- the diffusion time, $\tau_{\text {diff, }}$, from the place of generation to the space charge regions.

The drift transit time across the space charge regions can be expressed as:

$$
\tau_{d r}=\frac{w}{\mu_{a} E},
$$

where $w$ is the width of the space charge region, $\mu_{a}$ is the ambipolar carrier mobility, and denotes the mobility related to the move of carrier concentration distortion. The ambipolar carrier mobility can be expressed as: 


$$
\mu_{a}=\frac{n-p}{\frac{p}{\mu_{e}}+\frac{n}{\mu_{h}}} .
$$

If the generation takes place at a distance from the space charge region less than the ambipolar diffusion length $\left(l \ll L_{D}\right)$, the diffusion limited transit time, $\tau_{\text {diff, }}$ can be expressed by:

$$
\tau_{\text {diff }}=\frac{l^{2}}{2.4 D_{a}},
$$

where $D_{a}$ is the ambipolar diffusion coefficient:

$$
D_{a}=\frac{n+p}{\frac{p}{D_{e}}+\frac{n}{D_{h}}} .
$$

As we can see, the distribution of the electric field across the heterostructure may have an impact on carrier transport and device response. Figure 3 show the spatial distribution of the electric field for unbiased photodiodes with a constant and graded Cd alloy composition in the absorber layer, as well as for two doping levels. For the photodiode with a constant composition in the absorber region, the built-in electric field is mainly associated with the junction interfaces. For the grade structure, the electric field is build up in the entire absorber and is almost four orders of magnitude greater than for uniform structure. According to Eq. (11), increased electric field in the absorber region should improve the device response due to faster drift transport through this region. However, the time constant also depends on the doping level, due to the dependence of the ambipolar carrier mobility on carrier concentration.

Figure 4 shows spectral characteristics of normalized photocurrent $J(\omega) / J(0)$ for analysed devices with a low and high doping levels. The cut-off frequency $f_{T}$ is depicted by the - 3-dB line. Higher doping provides shifting the cut-off frequency towards higher values. This translates to several times shorter time constant. Example, for the device with a constant $\mathrm{Cd}$ alloy in the absorber layer, the time constant for a low doping level equals to

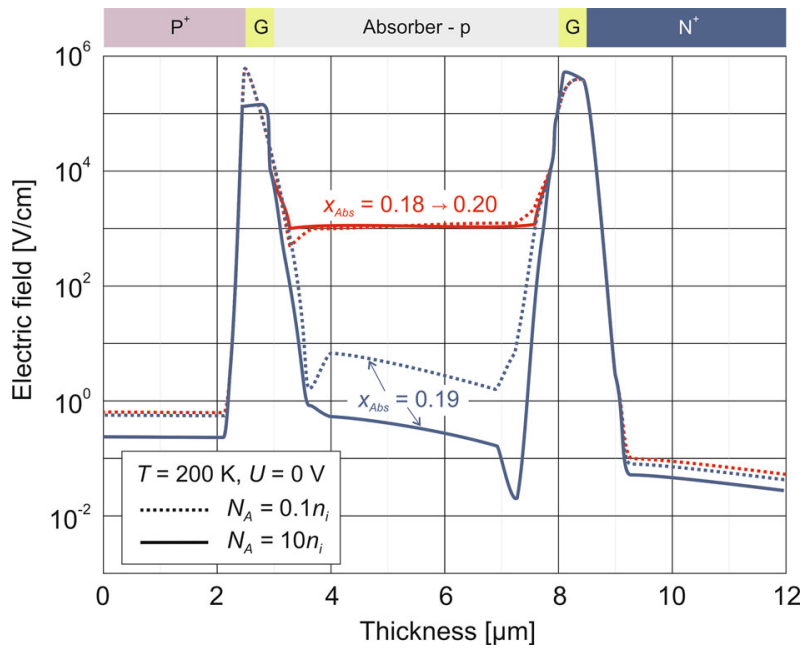

Fig. 3 Spatial distribution of the electric field for LWIR $\mathrm{N}^{+}-\mathrm{p}-\mathrm{P}^{+} \mathrm{HgCdTe}$ photodiodes 


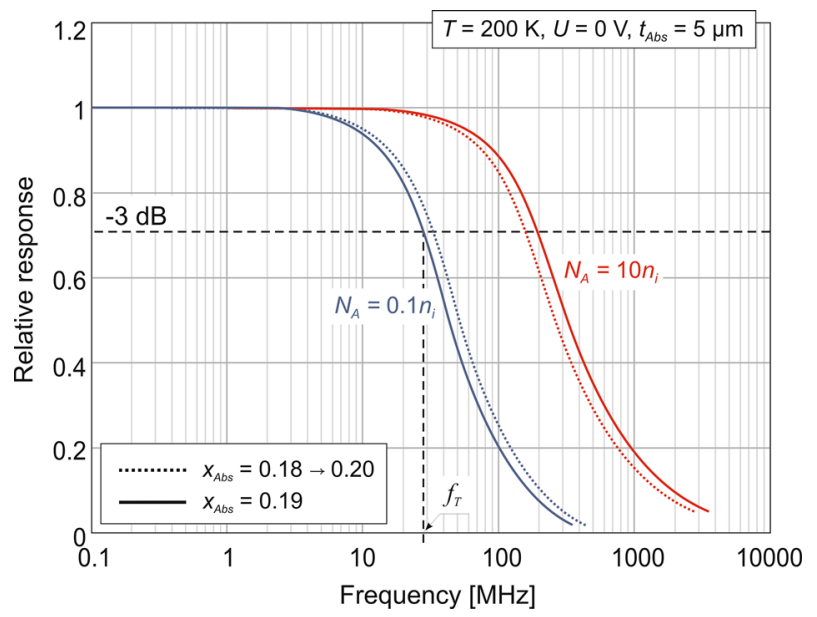

Fig. 4 Frequency response calculated for LWIR $\mathrm{N}^{+}-\mathrm{p}-\mathrm{P}^{+} \mathrm{HgCdTe}$ photodiodes

$5.6 \mathrm{~ns}$, while for a high doping level to $0.8 \mathrm{~ns}$. It is related to the values of the ambipolar diffusion coefficient (Fig. 5). For a high doping, the diffusion transit time across a $5 \mu \mathrm{m}$ thick absorber is of about 0.9 ns. The electric field built up in the junction interfaces slightly improves the response time, however, highly doped device is mainly limited to the diffusion time across the absorber region. The ambipolar diffusion coefficient is lower for the device with a graded Cd alloy composition in the absorber layer, therefore its time constant is longer. What more, larger electric field in the absorber region does not improve the response time of a grade device.

The situation is somewhat different for devices with a low doping level of the absorber. In this case, the diffusion transit time across a $5 \mu \mathrm{m}$ thick absorber is of about $15 \mathrm{~ns}$. The time constant on the level of $5 \mathrm{~ns}$ shows that it is a combination of the diffusion time and

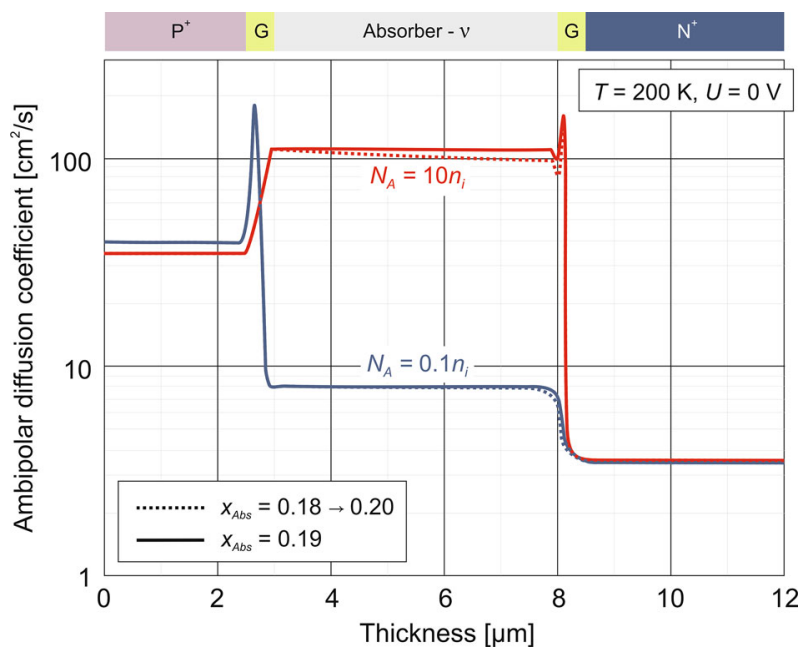

Fig. 5 Distribution of the ambipolar diffusion coefficient for LWIR $\mathrm{N}^{+}-\mathrm{p}-\mathrm{P}^{+} \mathrm{HgCdTe}$ photodiodes 
the drift time. Furthermore, the device with a graded composition achieves better response due to a higher ambipolar mobility in the absorber region (Fig. 6).

According to Eqs. (11) and (13), the time constant might be also improved by a thickness reduction the of the active region of the device. Figure 7 shows the time constant of analysed photodiodes as a function of the absorber doping, plotted for a two thickness values of the absorber layer, while Fig. 8 shows the time constant of analysed devices as a function of the absorber thickness, plotted for a two doping levels. Since a trade-off between contradictory requirements of reaching a fast response time and a high current responsivity is necessary, peak current responsivity has also been calculated. The time constant of the photodiodes assumes a constant values for the concentration of holes in the absorber up to the intrinsic concentration. Higher doping improves the time constant, as well as the current responsivity of the photodiodes.

As expected, the time constant of the photodiode decreases with a reduction of thickness of the absorption region. For $N_{A}=0.1 n_{i}$ doping level, the time constants below 1 ns can be obtained with thin absorption region. For high doping level $\left(N_{A}=10 n_{i}\right)$, the time constants below $1 \mathrm{~ns}$ is reachable for the absorber thickness less than $5 \mu \mathrm{m}$. Regardless of the doping level, the devices show a relatively low values of current responsivity for a $1-\mu \mathrm{m}$ thick absorber layer due to the only partial absorption of incoming photons. On the other hand, the absorption region cannot be too thick for the near-intrinsic material. In LWIR range, the minority carrier lifetime is quite short, mostly due to the Auger 1 process. The maximum current responsivity is reached by devices with $3-\mu \mathrm{m}$ thick absorber layer doped at a low level. For thicker absorbers, a significant portion of the carriers recombine before reaching the contacts. For higher doping, the current responsivity assumes almost constant values for absorbers thicker than $5 \mu \mathrm{m}$.

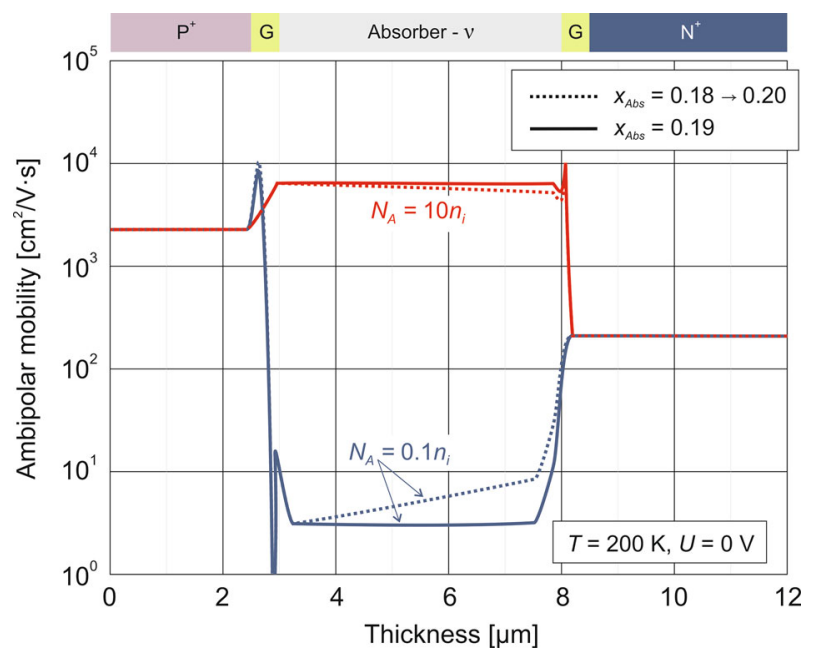

Fig. 6 Distribution of the ambipolar carrier mobility for LWIR ${ }^{+}-p-\mathrm{P}^{+} \mathrm{HgCdTe}$ photodiodes 


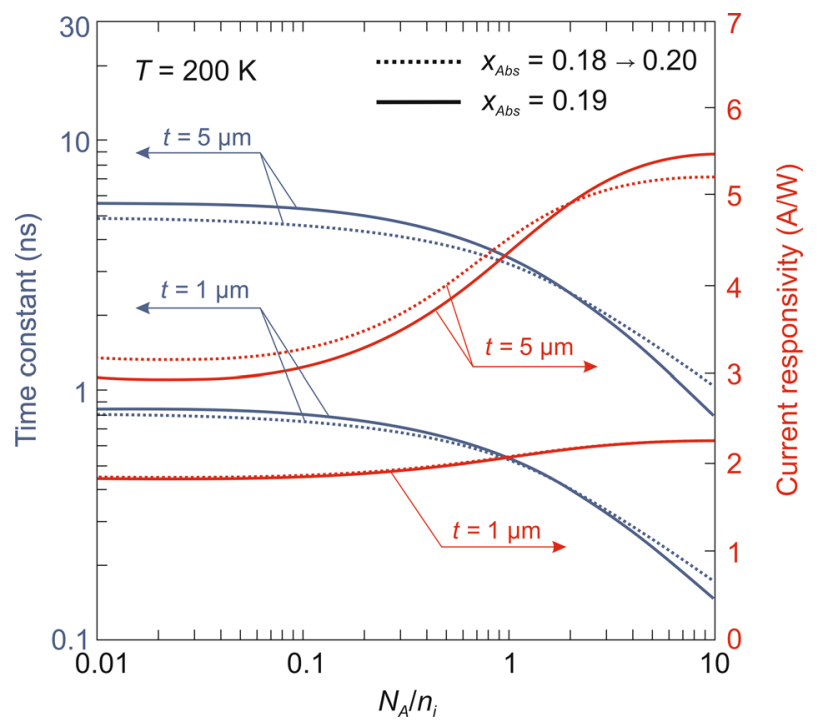

Fig. 7 Time constant and peak current responsivity of analyzed photodiodes as a function of absorber doping at zero bias operation

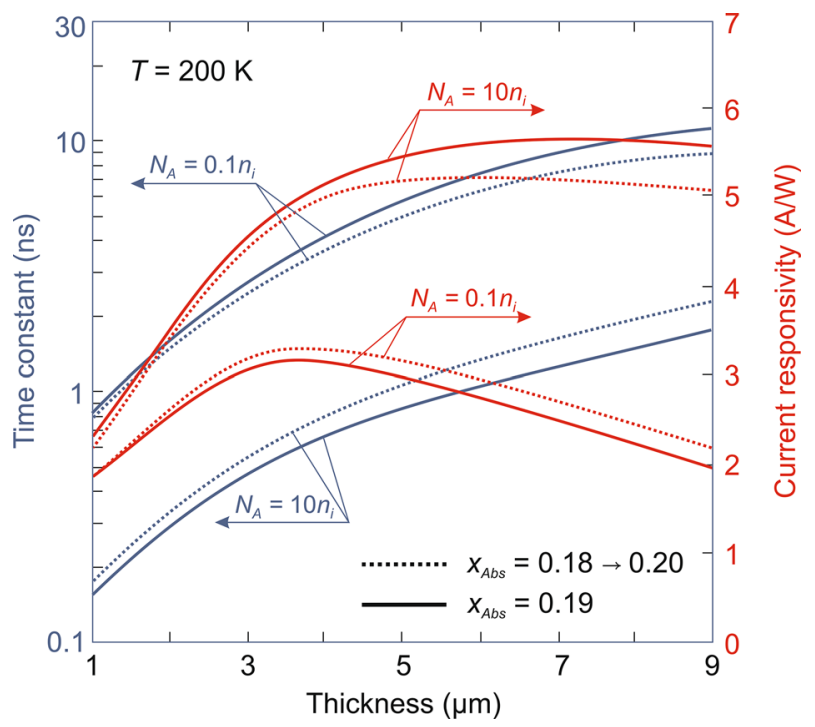

Fig. 8 Time constant and peak current responsivity of analyzed photodiodes as a function of absorber thickness at zero bias operation 


\section{Conclusions}

A formulation of carrier transport equations for $\mathrm{HgCdTe}$ heterostructure photodiodes is presented in Fourier space method in order to analyse spectrum characteristic of currents generated by harmonic optical signals. The method is valid in describing the high frequency response of LWIR photodiodes based on $\mathrm{HgCdTe}$ multilayer heterostructures.

In case of zero bias operation, the response time is mostly determined by an ambipoloar diffusion of photogenerated charge carriers to the junctions. The thinner absorption region, the faster photogenerated carriers reaching the junction. Therefore, reducing the thickness of the absorption region is good solution to improve the response speed of a detector. On the other hand, devices with too thin absorber would suffer from poor quantum efficiency and reduced responsivity.

Acknowledgements We acknowledge the support by The National Centre for Research and Development (Poland)—-the Grant No. TANGO1/2665576/NCBR/2015.

Open Access This article is distributed under the terms of the Creative Commons Attribution 4.0 International License (http://creativecommons.org/licenses/by/4.0/), which permits unrestricted use, distribution, and reproduction in any medium, provided you give appropriate credit to the original author(s) and the source, provide a link to the Creative Commons license, and indicate if changes were made.

\section{References}

Ashley, T., Elliott, C.T.: Nonequilibrium devices for infra-red detection. Electron. Lett. 21, 451-452 (1985)

Ashley, T., Elliott, T.C., White, A.M.: Infra-red detector using minority carrier exclusion. Proc. SPIE 572, 123-133 (1985)

Ciura, L., Kolek, A., Kłębowski, A., Stanaszek, D., Piotrowski, A., Gawron, W., Piotrowski, J.: Investigation of trap levels in $\mathrm{HgCdTe}$ IR detectors through low frequency noise spectroscopy. Semicond. Sci. Technol. 31, 035004-1-035004-7 (2016)

Elliott, C.T., Gordon, N.T., Hall, R.S., Phillips, T.J., White, A.M., Jones, C.L., Maxey, C.D., Metcalfe, N.E.: Recent results on metaloorganic vapor phase epitaxially grown $\mathrm{HgCdTe}$ heterostructure devices. J. Electron. Mater. 25, 1139-1145 (1996)

Emelie, P.Y., Philips, J.D., Velicu, S., Grein, C.H.: Modeling and design consideration of HgCdTe infrared photodiodes under nonequilibrium operation. J. Electron. Mater. 36(8), 846-851 (2007)

Emelie, P.Y., Velicu, S., Grein, C.H., Philips, J.D., Wijewarnasuriya, P.S., Dhar, N.K.: Modeling of LWIR $\mathrm{HgCdTe}$ Auger-suppressed infrared photodiodes under nonequilibrium operation. J. Electron. Mater. 37(9), 1362-1368 (2008)

Grodecki, K., Martyniuk, P., Kopytko, M., Kowalewski, A., Stępień, D., Kębłowski, A., Piotrowski, A., Piotrowski, J., Gawron, W., Rogalski, A.: Fast response HOT (111) HgCdTe MWIR detectors. Metrol. Meas. Syst. 24(3), 509-514 (2017)

Humpreys, R.G.: Radiative lifetime in semiconductors for infrared detectors. Infrared Phys. Technol. 26(1986), 337-342 (1986)

Jakšić, Z., Djurić, Z.: Extraction photodiodes with Auger suppression for all-weather free-space optical communication. Electronics 8(1), 30-32 (2004)

Jóźwikowska, A.: Numerical solution of the nonlinear Poisson equation for semiconductor devices by application of a diffusion-equation finite difference scheme. J. Appl. Phys. 104, 063715 (2008)

Jóźwikowski, K., Kopytko, M., Rogalski, A.: Numerical estimation of carrier generation-recombination processes and photon recycling effect in $3-\mu \mathrm{m}$ n-on-p $\mathrm{HgCdTe}$ photodiodes. Opt. Eng. 50, 061000-1-061000-8 (2011)

Jóźwikowski, K., Kopytko, M., Rogalski, A.: Numerical estimations of carrier generation-recombination processes and photon recycling effect in $\mathrm{HgCdTe}$ heterostructure photodiodes. J. Electron. Mater. 41, 2766-2774 (2012)

Kinch, M.A.: An infrared journey. Proc. SPIE 9451, 94512B (2015) 
Kopytko, M., Jóźwikowski, K., Rogalski, A., Jóźwikowska, A.: High frequency response of near-room temperature LWIR HgCdTe heterostructure photodiodes. Opto-Electron. Rev. 18, 277-283 (2010)

Kopytko, M., Jóźwikowski, K., Madejczyk, P., Pusz, W., Rogalski, A.: Analysis of the response time in high-temperature LWIR HgCdTe photodiodes operating in non-equilibrium mode. Infrared Phys. Technol. 61, 162-166 (2013)

Kopytko, M., Kębłowski, A., Gawron, W., Pusz, W.: LWIR HgCdTe barrier photodiode with Augersuppression. Semicond. Sci. Technol. 31, 035025 (2016)

Kurata, M.: Numerical Analysis of Semiconductor Devices. Lexington Books, DC Heath (1982)

Madejczyk, P., Gawron, W., Kębłowski, A., Martyniuk, P., Kopytko, M., Pusz, W., Stępień, D., Rutkowski, J., Piotrowski, A., Piotrowski, J., Rogalski, A.: Response time improvement of LWIR HOT MCT detectors. Proc. SPIE 10177, 1017719 (2017)

Martyniuk, P., Kopytko, M., Grodecki, K., Gawron, W., Gomółka, E.: High operating temperature longwave $\mathrm{HgCdTe}$ detector for fast response operation: optimization approach. Proc. SPIE 10034, 1003404 (2016)

Pawluczyk, J., Piotrowski, J., Pusz, W., Koźniewski, A., Orman, Z., Gawron, W., Piotrowski, A.: Complex behavior of time response of HgCdTe HOT photodetectors. J. Electron. Mater. 44, 3163-3173 (2015)

Piotrowski, J.: $\mathrm{Hg}_{1-\mathrm{x}} \mathrm{Cd}_{\mathrm{x}} \mathrm{Te}$ infrared photodetectors. In: Rogalski, A. (ed.) Infrared Photon Detectors, pp. 391-494. SPIE Press, Bellingham (1995)

Phillips, T.J., Gordon, N.T.: Negative diffusion capacitance in Auger-suppressed HgCdTe heterostructure diodes. J. Electron. Mater. 25(8), 1151-1156 (1996)

Piotrowski, J., Rogalski, A.: High-Operating-Temperature Infrared Photodetectors. SPIE, Bellingham (2007)

Piotrowski, J., et al.: Dark currents, responsivity, and response time in graded gap HgCdTe structures. Proc. SPIE 7660, 766031 (2010)

Van Roosbroeck, W.: Theory of the electrons and holes in germanium and other semiconductors. Bell Syst. Tech. J. 29, 560-607 (1950)

Velicu, S., Grein, C.H., Emelie, P.Y., Itsuno, A., Philips, J.D., Wijewarnasuriya, P.S.: MWIR and LWIR $\mathrm{HgCdTe}$ infrared detectors operated with reduced cooling requirements. J. Electron. Mater. 39(7), 873-881 (2010) 